\title{
Fratura Dentária em Crianças no Rio Grande do Sul: análise dos dados do levantamento SB-Gaúcho 2002/2003
}

\author{
Crown fractures in children in Rio Grande do Sul: data analysis of Survey SB-Gaúcho 2002/2003
}

\section{Marília Leão Goettems ${ }^{1}$, Eduardo Dickie de Castilhos ${ }^{2}$, Dione Dias Torriani ${ }^{3}$}

\begin{abstract}
Dental trauma is recognized as a public health problem due to the high frequency that occurs and the costs involved in treatment. Among the injuries, the crown fractures are one of the most frequent. The aim of this study was to describe the prevalence of crown fractures in children in the primary and permanent dentition in the state of Rio Grande do Sul. Secondary data from the Survey SB-Gaucho 2002/2003, of the State Department of Health were used. Data of 11,904 children were included, obtained with the diagnostic criteria of the World Health Organization. The prevalence of crown fracture at 18 to 36 months was $1.5 \%$, at 5 years was $1.8 \%$ and at 12 years was $2.7 \%$. It was found that dental trauma tended to increase with age, indicating that it would benefit from measures aimed at prevention of oral diseases. The index used possess limitations, which minimize dental trauma occurrence. Criteria including other types of dental trauma should be used by national surveys to obtain a more reliable estimate of its prevalence.
\end{abstract}

Keywords: Dentistry; Dental Health Surveys; Trauma.

\section{Resumo}

Os traumatismos dentários são reconhecidos como um problema de saúde pública devido à alta freqüência com que ocorrem e aos custos envolvidos no seu tratamento. Dentre os traumatismos, as fraturas dentárias são um dos tipos de maior ocorrência. O objetivo desse trabalho foi descrever a prevalência encontrada de fraturas dentárias em crianças na dentição decídua e permanente no estado do Rio Grande do Sul. Foram utilizados dados secundários de 11.904 crianças, avaliadas com os critérios de diagnóstico da Organização Mundial da Saúde, no levantamento SB-Gaúcho 2002/2003, da Secretaria Estadual de Saúde. Observou-se que dos 18 aos 36 meses a prevalência de fratura dentária foi de $1,5 \%$, aos 5 anos de $1,8 \%$ e aos 12 anos de 2,7\%. Apesar da limitação do critério diagnóstico utilizado, o qual minimiza a ocorrência do agravo, evidenciou-se que traumatismo dentário apresenta tendência de aumento com idade, indicando que se beneficiaria de medidas destinadas à prevenção. É importante que sejam utilizados nos levantamentos nacionais critérios que inclúam outros tipos de traumatismos dentários, visando obter-se uma estimativa mais confiável de sua ocorrência.

Palavras- chave: Odontologia; Levantamentos de saúde bucal; Traumatismos.
${ }^{1}$ Cirurgiã-dentista, Mestre em Odontopediatria, Programa de Pós-graduação em Odontologia da Universidade Federal de Pelotas

${ }^{2}$ Cirurgião-dentista, Mestre em Odontologia pela Universidade Federal do Rio Grande do Sul e Doutorando em Epidemiologia pela Universidade Federal de Pelotas, Professor do Departamento de Odontologia Social e Preventiva da Faculdade de Odontologia da Universidade Federal de Pelotas

${ }^{3}$ Mestre e Doutora em Odontopediatria pela Universidade Estadual de São Paulo, Professora da Faculdade de Odontologia e do Programa de Pós-graduação em Odontologia da Universidade Federal de Pelotas

Correspondência: Dione Dias Torriani

Endereço: Faculdade de Odontologia. Rua Gonçalves Chaves, 457 - CEP 96015-560, Pelotas - RS,Brasil

Fone: 53 3222-6690

E-mail: dionedt@gmail.com

Data de Submissão: 24/02/2010

Data de Aceite: 14/05/2010

\section{Introdução}

As injúrias dentárias traumáticas podem ser causadas por impactos acidentais ou agressões físicas e, dependendo de sua intensidade e extensão, afetam os tecidos mineralizados, 0 processo alveolar e o periodonto (FELICIANO; FRANÇA CALDAS JUNIOR, 2006). Estudos epidemiológicos relatam prevalências que variam desde 10 a $36 \%$ na dentição decídua (MESTRINHO BEZERRA; CARVALHO, 1998; WENDT et al., 2010) e de 2,4 a $36,1 \%$ (GRIMM, et al., 2004; CAVALCANTI et al., 2009) na dentição permanente. Os resultados variam de acordo com a metodologia e os critérios utilizados e também em diferentes populações e idades. Dentre os estudos de base populacional, é consenso que as fraturas coronárias são as injúrias mais prevalentes, tendo sido constatado que a grande maioria permanece sem o tratamento adequado (FERGUSON; RIPA,1979; GARCIA-GODOY et al., 1983; YAGOT; NAZHAT; KUDER et al., 1988; OTUYEMI; SEGUN-OJO; ADEGBOY, 1996; MESTRINHO; BEZERRA; CARVALHO, 1998; HARGREAVES et al., 1999; KRAMER et al., 2003; GRANVILLE-GARCIA; MENEZES; LIRA, 2006).

Andreasen e Andreasen (1994) já relatavam que o aumento da violência, dos acidentes de trânsito e da participação das crianças e adolescentes em esportes de contato tendem a causar o aumento da importância dos traumatismos dentários em algumas regiões, com consequências econômicas significativas (ANDREASEN; ANDREASEN, 1994).

Dessa forma, devido à alta frequência em que ocorrem, especialmente em crianças pequenas, e também pelos custos e complexidade do tratamento muitas vezes requerido, os traumatismos dentários são reconhecidos como um problema de saúde pública (GLENDOR, 2008).

Estudos epidemiológicos representativos da população provêm dados importantes acerca da ocorrência de traumatismos, fornecendo um real dimensionamento do problema. O Ministério da Saúde, com o objetivo de avaliar as condições de saúde bucal da população e produzir informações para subsidiar o planejamento e avaliação das ações nos diferentes níveis de gestão do SUS, financiou o levantamento epidemiológico SB-Brasil 2002-2003. Neste estudo, foram avaliadas diferentes condições de saúde bucal de 108.921 brasileiros de diferentes grupos etários, residentes nas cinco macrorregiões do país (BRASIL, 2004). 
Apesar de sua relevância, os dados quanto à presença de dentes fraturados não foram, até o presente momento, divulgados pelo Ministério da Saúde. Dessa forma, o objetivo desse trabalho foi descrever a prevalência encontrada de fraturas dentárias em crianças na dentição decídua (faixa etária de 18-36 meses e aos 5 anos) e permanente (aos 12 anos) no estado do Rio Grande do Sul.

\section{Metodologia}

Foram utilizados dados secundários, oriundos do Projeto SB Brasil 2003 - Condições de Saúde Bucal da População Brasileira (BRASIL, 2004), um estudo transversal realizado pelo Ministério da Saúde entre maio de 2002 e outubro de 2003. Tal projeto foi aprovado pelo Conselho Nacional de Ética em Pesquisas com Seres Humanos (CONEP), sob o parecer de número 581/2000 e todos os indivíduos assinaram o termo de consentimento.

A técnica de amostragem probabilística por conglomerados em múltiplos estágios permitiu a produção de inferências para cada uma das macrorregiões brasileiras, por porte do município e para cada idade ou grupo etário. Foram avaliados indivíduos de 18 a 36 meses, 5, 12 anos, 15 a 19, 35 a 44 e 65 a 74 anos.

No estado do Rio Grande do Sul, foram coletados dados de 34.472 pessoas nos 7 pólos regionais do estado (Sul, Serra, Norte, Missioneira, Vales, Metropolitana e Centro-oeste), a fim de garantir representatividade de todas as regiões. Do total, 7.755 eram crianças pré-escolares, sendo 3.427 da faixa etária de 18 a 36 meses e 4.328 com 5 anos de idade. Aos 12 anos foram avaliadas 5.018 crianças. De acordo com o relatório final do SB-RS, o delineamento assegura a produção de inferências para cada uma das 7 macrorregiões do estado e para cada idade ou grupo etário (RIO GRANDE DO SUL, 2003).

A coleta de dados foi realizada por cirurgiões-dentistas previamente treinados nos domicílios, creches e escolas, de acordo com o grupo etário. Foram incluídas todas as crianças presentes nos locais sorteados, após assinatura do termo de consentimento pelos responsáveis.

A condição da coroa dentária foi avaliada pelo código $T$ do índice CPO-D, da Organização Mundial da Saúde (OMS). Tal índice inclui nos seus critérios a presença de fratura dentária. Segundo a OMS a coroa é considerada fraturada quando parte de sua superfície está ausente como resultado de um traumatismo, mas sem evidência de cáries (WORLD HEALTH ORGANIZATION, 1987). Para realização deste trabalho, foi criada a variável prevalência de traumatismo, dicotomizada em fratura presente ou ausente.

Foi feita a análise descritiva dos dados para as 7 macrorregiões do estado, de acordo com a idade ou faixa etária (18-36 meses, 5 e 12 anos).

\section{Resultados}

A tabela 1 mostra as prevalências encontradas de traumatismo e de cárie nos diferentes grupos etários avaliados. Dados da região centro-oeste (869 crianças) não puderam ser avaliados, por estarem indisponíveis. Dessa forma, dados quanto à presença de fratura dentária de 11.904 crianças foram obtidos.

A prevalência de fratura dentária em crianças no estado foi $2,1 \%$, sendo de $1,5 \%$ em crianças de 18 a 36 meses, $1,8 \%$ aos 5 anos e 2,7\% aos 12 anos, na dentição permanente.
Tabela 1. Número e percentual de crianças segundo presença de fratura dentária na faixa etária de 18 a 36 meses, aos 5 anos e aos 12 anos, nas diferentes macrorregiões do Rio Grande do Sul, 2003.

\begin{tabular}{|c|c|c|c|}
\hline Região & Idade & $\begin{array}{l}\text { Fratura Presente } \\
\mathrm{n}(\%)\end{array}$ & Total \\
\hline \multirow{3}{*}{ Sul } & $18-36$ meses & $4(1,3)$ & 305 \\
\hline & 5 anos & $2(0,8)$ & 260 \\
\hline & 12 anos & $4(1,0)$ & 390 \\
\hline \multirow{3}{*}{ Serra } & $18-36$ meses & $3(0,9)$ & 348 \\
\hline & 5 anos & $3(0,6)$ & 525 \\
\hline & 12 anos & $11(1,9)$ & 575 \\
\hline \multirow{3}{*}{ Norte } & $18-36$ meses & $8(1,0)$ & 755 \\
\hline & 5 anos & $11(2,0)$ & 562 \\
\hline & 12 anos & $5(0,9)$ & 573 \\
\hline \multirow{3}{*}{ Missioneira } & $18-36$ meses & $4(0,7)$ & 555 \\
\hline & 5 anos & $7(1,2)$ & 593 \\
\hline & 12 anos & $7(1,2)$ & 597 \\
\hline \multirow{3}{*}{ Metropolitana } & $18-36$ meses & $14(2,1)$ & 660 \\
\hline & 5 anos & $43(2,5)$ & 1747 \\
\hline & 12 anos & $80(3,7)$ & 2174 \\
\hline \multirow{3}{*}{ Vales } & $18-36$ meses & $13(3,5)$ & 375 \\
\hline & 5 anos & $8(1,9)$ & 420 \\
\hline & 12 anos & $20(4,0)$ & 490 \\
\hline \multirow{3}{*}{$\begin{array}{l}\text { Centro- } \\
\text { Oeste }\end{array}$} & $18-36$ meses & $\ldots$ & 429 \\
\hline & 5 anos & $\ldots$ & 221 \\
\hline & 12 anos & $\ldots$ & 219 \\
\hline \multirow[t]{3}{*}{ RS } & $18-36$ meses & (46) 1,5 & 2998 \\
\hline & 5 anos & (74) 1,8 & 4107 \\
\hline & 12 anos & (127) 2,7 & 4799 \\
\hline
\end{tabular}

... 429, 221 e 219 dados indisponíveis para 18-36 meses, 5 e 12 anos, respectivamente

A figura 1 mostra a distribuição geográfica segundo a média estadual de prevalência de fratura dentária. Para todas as faixas etárias o mapa apresentou distribuição similar, sendo que as macrorregiões dos Vales e Metropolitana apresentaram prevalências acima da média estadual.

$\square$ Dados não disponiveis

$\square \leq$ média estadual

$\square$ Acima da média estadual

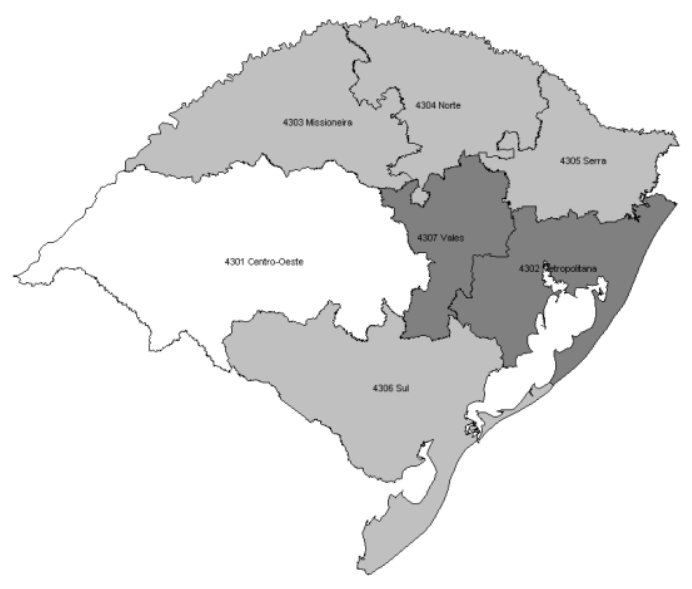

Figura 1. Distribuição da ocorrência de fratura dentária em crianças nas macrorregiões do Rio Grande do Sul, 2003. 


\section{Discussão}

Podem ser apontados como pontos positivos deste estudo - tamanho da amostra e a representatividade populacional alcançada (BORGES et al., 2008). O critério utilizado para avaliação de traumatismo dentário apresenta limitações que devem ser ressaltadas. $O$ índice inclui apenas as fraturas dentárias, um dos tipos de traumatismos e, de acordo com as orientações da OMS, dentes com traumatismo e cárie são classificados apenas como cariados e dentes com fraturas tratadas por restaurações são considerados como restaurados. Além de subestimar a ocorrência, o critério também não permite a classificação do traumatismo de acordo com sua severidade.

A prevalência de traumatismo dentário em estudos brasileiros é de aproximadamente $16 \%$ para crianças até 36 meses (CUNHA; PUGLIESI; MELLO VIEIRA, 2001), 30\% até 5 anos (MESTRINHO; BEZERRA; CARVALHO, 1998; KRAMER et al., 2003; WENDT et al., 2010) e varia até 36,1\% (CAVALCANTI et al., 2009) aos 12 anos. Na maioria dos estudos, a fratura é citada como o tipo de traumatismo mais prevalente. Cunha, Pugliesi e Mello Vieira (2001) encontraram $48,4 \%$ dos traumatismos como sendo fraturas não complicadas de coroa até os 3 anos de idade. Em outra pesquisa avaliando a dentição permanente, ao redor de $70 \%$ dos traumatismos dentários foram diagnosticados como sendo fraturas (CAVALCANTI et al., 2009). Entretanto, a comparação com esses resultados torna-se difícil, devido aos diferentes critérios e metodologias empregadas.

Utilizando-se do mesmo critério deste estudo, embora acrescentando às fraturas os dentes perdidos por motivos que não cárie, a prevalência de traumatismo em dentes anteriores foi de 2,4\% em crianças de 5 a 12 anos de idade do estado de São Paulo (GRIMM et al., 2004). Os autores também avaliaram que o método utilizado minimiza a ocorrência de traumatismo. Na presente avaliação, por todos os dentes terem sido incluídos, não pareceu adequado incluir o critério dentes perdidos por motivos que não cárie, pois dentes ausentes por motivos periodontais, ortodônticos ou congênitos poderiam ser erroneamente classificados como perdas por traumatismo.

Apesar das limitações do instrumento de medida, observou-se na dentição decídua uma tendência de a prevalência de traumatismo aumentar com a idade. Entretanto esse dado deve ser analisado com cautela, pois há o efeito cumulativo das injúrias, especialmente em tecidos duros, podendo o aumento não estar relacionado com uma maior ocorrência de acidentes em crianças maiores (FORSBERG; TEDESTAM, 1993; KRAMER et al., 2003). De acordo com a literatura, as crianças pequenas estariam mais sujeitas a fatores de risco aos traumatismos, por estarem aprendendo a caminhar e explorar os ambientes, sendo as quedas ocorrências comuns (JOHO; MARECHAUX, 1980; CUNHA; PUGLIESI; MELLO VIEIRA, 2001). A despeito de não ser um estudo longitudinal, os dados sugerem que cerca de $83 \%$ dos traumatismos ocorreram até os 3 anos de idade. Isso demonstra a importância de que os cirurgiões-dentistas e demais profissionais da saúde informem os pais sobre a medidas de prevenção às injúrias dentárias acidentais o mais precocemente possível (ROBSON et al., 2009). Na dentição permanente observou-se a maior ocorrência, denotando a necessidade de estender a atenção a este agravo em crianças escolares.

Uma forma de minimizar possíveis seqüelas decorrentes de traumatismo dentário, bem como de realizar-se abordagem preventiva em relação aos acidentes seria a realização da primeira visita ao dentista dentro do primeiro ano de vida da criança, conforme recomendado pela literatura. Entretanto, como no Brasil apenas uma pequena parcela das crianças tem acesso ao serviço odontológico na infância (BARROS; BERTOLDI, 2002), outras formas de acesso à informação devem ser estabelecidas. Programas preventivos dirigidos a professores e pais, além de campanhas informativas, são citados como alternativas para aumentar o conhecimento sobre injúrias traumáticas e sobre a importância de procurar atendimento odontológico após sua ocorrência (CAVALCANTI et al., 2009). No âmbito da Estratégia de Saúde da Família, é reforçado o papel do agente comunitário de saúde na promoção de saúde bucal e também para aumentar o acesso e uso de serviços odontológicos (FRAZAO; MARQUES, 2008).

A prevalência de trauma observada em crianças no estado do Rio Grande do Sul foi de 2,1\%. Entretanto, provavelmente essa prevalência seja maior que a encontrada, devido às características já citadas do instrumento utilizado para a coleta de dados. Com relação às macrorregiões do estado, a prevalência foi acima da média para todas as idades na região dos Vales e Metropolitana. É possível que isso resulte de diferenças no processo de treinamento e calibração, em que mais ênfase no diagnóstico de fraturas durante esse processo tenha sido dada nessas regiões e, ainda, como a fratura é um código incluído no índice cujo objetivo principal é avaliar cárie dentária, seria esperado que maior treinamento fosse dispensado ao diagnostico de cárie que de fratura.

Para aumentar o conhecimento sobre a epidemiologia dos traumatismos dentários, é importante que além dos problemas já avaliados - condição da coroa, doença periodontal, fluorose, máoclusão e prótese - os próximo levantamentos nacionais de saúde bucal incluam um exame de traumatismo que forneça mais informações sobre sua ocorrência e severidade. A partir desses resultados, estratégias preventivas poderão ser desenvolvidas e implementadas, visando reduzir a prevalência e também custos envolvidos no tratamento de injúrias dentárias (CAVALCANTI et al., 2009). Corroborando com tais achados, na divulgação da Proposta de Projeto Técnico para Consulta Pública do Levantamento SBBrasil 2010 (BRASIL, 2009) afirmou-se ser importante que o traumatismo seja avaliado de forma individual. Dessa forma, durante a coleta de dados, realizada no primeiro semestre de 2010 os dentes permanentes anteriores serão classificados segundo a presença e severidade de fratura dentária e presença de avulsões.

\section{Conclusões}

Os traumatismos configuram-se como um problema de saúde pública, pela ocorrência relativamente alta, pelas consequências originadas e pela possibilidade de serem prevenidos, justificando sua atenção dentro da Estratégia de Saúde da Família;

Enfatiza-se a importância de se divulgarem os dados obtidos nesse levantamento nacional, inclusive os dados de fratura dentária, dada a representatividade da população estudada. Mais pesquisas são necessárias em nível nacional utilizando critérios mais específicos para avaliar a ocorrência e severidade de traumatismos.

\section{Referências}

ANDREASEN, J.O.; ANDREASEN, F.M. Textbook and color atlas of traumatic injuries to the teeth. $3^{\text {rd }}$ ed. Copenhagen: Munksgaard, 1994. $771 \mathrm{p}$.

BARROS, A.J.D.; BERTOLDI, A.D. Desigualdades na utilização e no acesso a serviços odontológicos: uma avaliação em nível nacional. Ciência Saúde Coletiva, Rio de Janeiro, v. 7, n. 4, p. 709-717, 2002.

BORGES, C. M. et al. Dental and gingival pain and associated factors among Brazilian adolescents: an analysis of the Brazilian Oral Health Survey 2002-2003. Cad. Saúde Públ., Rio de Janeiro, v. 24 , n. 8 , p. $1825-1834$, Aug. 2008

BRASIL. Ministério da Saúde. Projeto SB Brasil 2003. Condições de saúde bucal da população brasileira: 2002-2003. Resultados principais._Brasília, 2004 
Proposta de projeto técnico para consulta pública do levantamento SB-Brasil 2010.Brasília, 2009.

CAVALCANTI, A. L. et al. Traumatic anterior dental injuries in 7- to 12-year-old Brazilian children. Dent. Traumatol., Copenhagen, v. 25, no. 2, p. 198-202, Apr. 2009.

CUNHA, R. F.; PUGLIESI, D.M.; MELLO VIEIRA, A.E. de . Oral trauma in Brazilian patients aged 0-3 years. Dent. Traumatol., Copenhagen, v.17, no. 5, p.210-212, Oct. 2001.

FELICIANO, K. M.; FRANÇA CALDAS JUNIOR, A. de. A systematic review of the diagnostic classifications of traumatic dental injuries. Dent. Traumatol., Copenhagen, v. 22, no. 2, p.7176, Apr. 2006.

FERGUSON, F.S.; RIPA, L.W. Prevalence and type of traumatic injuries to the anterior teeth of preschool children. J. Pedod., Boston, v. 4, no.1, p.3-8, Fall 1979.

FORSBERG, C.M.; TEDESTAM, G. Etiological and predisposing factors related to traumatic injuries to permanent teeth. Swed. Dent. J., Jonkoping, v. 17, no. 5, p.183-190, 1993.

FRAZAO, P.; MARQUES, D. Efetividade de programa de agentes comunitários na promoção da saúde bucal. R. Saúde Publ., São Paulo, v. 43, n. 3, maio/jun. 2009.

GARCIA-GODOY, $F$. et al. Traumatic dental injuries in preschoolchildren from Santo Domingo. Community Dent. Oral Epidemiol., Copenhagen, v. 11, no. 2, p.127-130, Apr. 1983.

GLENDOR, U. Epidemiology of traumatic dental injuries--a 12 year review of the literature. Dent. Traumatol., Copenhagen, v. 24, no. 6, p.603-611, Dec. 2008.

GRANVILLE-GARCIA, A. F.; MENEZES, V.A. de; LIRA, P.I.C. Dental trauma and associated factors in Brazilian preschoolers. Dent. Traumatol., Copenhagen, v. 22, no. 6, p. 318-322, Dec. 2006.

GRIMM, S., P. et al. Dental injury among Brazilian schoolchildren in the state of Sao Paulo. Dent. Traumatol., Copenhagen, v. 20, no. 3, p.134-138, June 2004.

HARGREAVES, J. A. et al. Trauma to primary teeth of South African pre-school children. Endod. Dent. Traumatol., Copenhagen, v.15, no. 2, p.73-76, Apr. 1999.

JOHO, J.P.; MARECHAUX, S.C. Trauma in the primary dentition: a clinical presentation. ASDC J. Dent. Child, Chicago, v. 47, no. 3 , p.167-174, May/June 1980.

KRAMER, P.F. et al. Traumatic dental injuries in Brazilian preschool children. Dent. Traumatol., Copenhagen, v. 19, no. 6, p. 299-303, Dec. 2003.

MESTRINHO, H.D.; BEZERRA, A.C.; CARVALHO, J.C. Traumatic dental injuries in Brazilian pre-school children. Braz. Dent. J., Ribeirao Preto, v. 9, no. 2, p.101-114, 1998.

OTUYEMI, O.D.; SEGUN-OJO, I.O.; ADEGBOYE, A.A. Traumatic dental injuries in Nigerian preschool children. East Afr. Med. J., Kenya, v. 73, no. 2, p. 604-606, Sept. 1996.

ROBSON, F. et al. Prevalence and determining factors of traumatic injuries to primary teeth in preschool children. Dent. Traumatol., Copenhagen, v. 25, no. 1, p.118-122, Feb. 2009.
RIO GRANDE DO SUL. Secretaria da Saúde. Seção de Saúde Bucal. Projeto SB/RS Condições de saúde bucal da população do Rio Grande do Sul. Porto Alegre, 2003.

WENDT, F. P. et al. Traumatic dental injuries in primary dentition: epidemiological study among preschool children in South Brazil. Dent. Traumatol., Copenhagen, v. 26, no. 2, p.168-173, Apr. 2010.

WORLD HEALTH ORGANIZATION. Oral health surveys: basic methods. Geneva, 1987.

YAGOT, K. H.; NAZHAT, N.Y.; KUDER, S.A. Traumatic dental injuries in nursey schoolchildren from Baghdad, Iraq. Community Dent. Oral Epidemiol., Copenhagen, v. 39, no. 5, p.133-167, Oct. 1988. 